\title{
Networked Systems : Introducing Network Management Models into ODP
}

\author{
Simon ZNATY ${ }^{1}$, Guy GENILLOUD ${ }^{2}$, Jean-Paul GASPOZ ${ }^{1}$, Jean-Pierre HUBAUX ${ }^{1}$ \\ Swiss Federal Institute of Technology \\ 1 Telecommunications Laboratory, ${ }^{2}$ Industrial Computer Engineering Laboratory \\ CH-1015 Lausanne, Switzerland.
}

\begin{abstract}
The complexity of networked systems calls for their management. The ODP framework based on viewpoints provides powerful concepts to support the specification and design of distributed systems, but without fully considering their management. In order for ODP to avoid re-inventing the wheel with regard to management, the results of network management standards that we have structured in terms of models should be integrated in the ODP framework. Therefore, this paper defines the mapping between the network management models and the ODP viewpoints. Emphasis lies on the realization of a connection management function using the proposed method.
\end{abstract}

\section{Introduction}

Networked systems are becoming large, complex and heterogeneous. Moreover they include both communication and information processing. Such a scenario gives rise to several requirements relating to management of these distributed systems. Indeed, to provide their services efficiently, networked systems require management tools to control and maintain in a consistent and a uniform way the largc number of resources attached to them. These include network, system and application resources.

Our study of network management, based on work done by standard bodies, shows that five network management models are necessary to fully cover all network management requirements. These are the information, functional, architectural, communication and organizational models [1]. The information model reprcsents the network by management information. Management information means the information that is used to define how to manage particular types of resources. The functional model defines the management functions to be implemented to reach the management objectives. The architectural model describes the internal structure of the entities performing the management tasks and their interfaces. The communication model enables exchanges of management information between management entities. The organizational model defines how management entities are organized and what are their interrelations. These models are generic enough to be applicable to the management of networked systems.

To support the specification and design of distributed systems, the ODP (Open Distributed Processing) standards [2][3][4] provide a reference model and powerful concepts. Moreover, the use of five different viewpoints advocated by ODP, each viewpoint representing a different abstraction of the original system, allows the inherent complexity of distributed systems to be reduced. These are the enterprise, information, computation, engineering and technology viewpoints.

An issue is that ODP will need to include the management of distributed systems. To do so, it is necessary to describe the network management models using ODP viewpoints, in order for ODP to reuse work already done on systems management. Therefore, the proposed approach to develop the management of distributed systems as well as distributed management systems, is to define the mapping between network management models and ODP viewpoints. Since networked systems management is handled using our models, their integration within ODP is easy.

In section 2, our network management models and the ODP viewpoints are introduced. In section 3 , the network management models are described using the ODP viewpoints; the goal is to specialize ODP to Open Distributed Management. Since ODP is not very prescriptive regarding the scope of each viewpoint as well as the techniques / languages used in each viewpoint, this section also focuses on the techniques applied at each viewpoint for the specification of Open Distributed Management. These are based on the notations proposed in OSI management standards. Section 4 focuses on the application of our concepts to the realization of a connection management function.

\section{Network Management Models and ODP Viewpoints}

\section{II.1. Network Management Models}

To deal with all network management requirements and complexities, the definition of network management viewpoints or models is necessary. A network management model represents a network management system with emphasis on a specific concern. Network management involves the network representation by management information, the protocol to access and manage these information, the management functions to fulfil the management objectives and the management entities to guarantee an operational network. From this definition, five network management models are proposed, namely functional, organizational, information, architectural, and communication models [1].

These viewpoints have been obtained through the study and reuse of the work done by the current network management standardization bodies. These are ISO concentrating on how to manage Open Systems Interconnection (OSI) networks [5], ITU concerned with how to manage telecommunication networks [6], the NM Forum focusing on the integration of the management of OSI, telecommunications and Internet worlds [7], IETF dealing with TCP/IP based networks management [8] and TINA-C [9] concentrating on the integration of the IN (Intelligent Network) and TMN (Telecommuniciations Management Network) architectures. Our proposed five network management models derived from the above standards are detailed hereafter.

\section{II.1.1. Functional Model}

The management objectives should be defined in terms of management functions. For this purpose, the ISO has introduced five management functional areas (Configuration, Performance, Fault, Security and Accounting management) [10]. These are applicable to both networks and information processing systems. The set of functional areas selected for the management of a given nctworked system configuration constitutes the functional model. These are the important areas of interest to enterprises wishing to employ systems management.

\section{II.1.2. Organizational Model}

The organizational model defines the management organization in terms of managers and agents, where these are placed, and what are their interactions. The terms managers and agents come from ISO management standardization [5]. However these standards do not require that these management entities be placed at a particular location in the networked system. The organizational model is derived from the architecture of the networked system to be managed. Some rules are already well established for the location of the management entities. For instance, agents are usually placed in components such as servers, gateways, bridges and routers while network control stations are typically managers. 


\section{II.1.3. Information Model}

The information model represents the networked system by management information. Representing this information first requires modeling those aspects of the resource that are of interest to management. The main contribution in this area comes from ISO. The OSI systems management information model [11] has emerged as an object oriented paradigm for the modcling of systems and network resources for use in management. The OSI information model is object oriented [11], i.e., it is characterized by the definition of objects with specified properties (managed objects) and relationships between these objects.

The OSI information model has found acceptance within ITU, TINA, NMForum and ETSI standards bodies for the modeling of network equipments, networks and services. ITU [12], ETSI [13] and TINA-C [14] propose generic information models to be reused for the management of any typc of telecommunications network, e.g., ATM, SDH, PDH, etc. On the contrary, the Internet standard framework (IETF), views managed objects as little more than simple (scalar) variables residing in a virtual store (MIB II [15]).

\subsubsection{Architectural Model}

The Architectural model describes the internal structure of the entities performing the management tasks, and their interfaces. These entities are identified earlier in the organizational model. Every network managcment standardization body defines a management architecture, but the most advanced is the TMN proposed by ITU. TMN is a set of standards that define an architecture for a telecommunication Management Network [6] and is the basis for our architectural model. TMN provides three kinds of architectures, the information architecture (i.e., the information model), functional architecture (function blocks and reference points between these), and physical architecture (implementation of the TMN architecture consisting of physical elements).

Moreover four levels of operations and management are defined within TMN in the information architecture part: network clcment level, network level, service level and business level. All these management levels are generic with regard to technology and implementation and numerous capabilities are needed to achieve full management at each and every level. These levels are those where the information models take place. We call these four layers the information architectural model.

The main component of the architectural model is the TMN functional architecture that we name the functional architectural model. This latter is described in terms of function blocks and reference points between these. The function blocks provide the TMN general functions which enable a TMN system to perform the TMN application functions. Reference points are conceptual points of information exchange between non overlapping function blocks. A reference point becomes an interface when the connected function blocks are embodied in separate pieces of equipment.

The physical architectural model specifies the realization of I'MN.

To summarize, the functional architectural model describes the internal structure of function blocks and the reference points between these function blocks, the information architectural model defines the management layers where function blocks encapsulating management information are present, and the physical architectural model defines the physical entities and their interfaces. Note that the architectural model is general enough to be applicable to any type of networked system.

\section{II.1.5. Communication Model}

Communications mechanisms to distribute and coordinate management tasks between different management systems are required. This aspect is within the scope of the communication model, i.e., a uniform communications platform to support management exchanges between management entities. For systems management, two main network management protocols bave becn proposed. These are the OSI standard CMIP (Common Management Information Protocol) [16], and the IETF standards SNMP (Simple Network Management Protocol) [17] with its enbanced version SNMPv2 [18].

II.1.6. The Network Management Models within the Software Development Process

The different models advocated by network management are ordered and categorized according to the steps of the software development process, namely requirements, analysis, design and implementation (Figure 1). This is a natural approach and method for the realization of a network management system. The requirements represent a detailed refinement of the system objcctivc. The goal of the analysis step is to develop a model of what the system will do, so that it can be understood. During design, decisions are made about how the problem will be solved, first at a high level (bigh level design) where the overall architecture is built, then at increasing detailed levels (detailed design). During design, the focus is on how the system is done. The implementation step covers code writing that should be a simple translation of the design decisions into the peculiarities of a particular programming language.

The functionalities of the different models or model components present at each step are outlined hereafter:

1. Identification of the management laycrs (subset of the TMN layers) where management information should take place.

2. Definition of the management organization within the management layers and between these layers.

3. Definition of the management objectives in terms of managcment functional areas to be tackled for enterprise systems management. Every management function involved in the functional model is specified according to a set of policies.

4. Specification of an information model using the results of phases 1, 2, and 3. An information model is present at every management layer for each management entity.

5. Design of the overall management architectural model in terms of function blocks and reference points between these function blocks. This model reuses the result of phase 4 since function blocks encapsulate management information.

6. Detailed design of the abstract mechanisms necessary for running the architectural model.

7. \& 8. Implementation of the architectural model (physical management blocks) and the interactions between these blocks using a specific management protocol.

\section{II.2. The ODP Viewpoints}

Open distributed Processing (ODP) is a major ISO research and standardization initiative aimed at developing distributed processing systems based on open standards. The scope of ODP encompasses all potential applications of distributed processing systems, including telecommunications services, networks and their support environments. To deal with the complexity of an ODP system, the system is considered from different viewpoints. Each viewpoint represents a different abstraction of the original system. Informally, a viewpoint leads to a representation of the system with emphasis on a specific concern. Five viewpoints arc identificd: cntcrprise, information, computation, engineering and technology.

The enterprise viewpoint is concerned with the overall environment within which an ODP system is to operate. The information viewpoint focuses on the information requirements of the system, and deals with information object types, together with their states and permitted state changes. The computation viewpoint shows processing functions and data types, abstracting away from the underlying hardware structures via transparency functions. The engineering viewpoint establishes transparency services utilizing concepts from operating systems and 
communications. The technology viewpoint is concerned with the realization of an ODP system in terms of specific hardware and software components.

II.2.1. The ODP Vicwpoints within the Software Development Process

Although the reference model for Open Distributed Processing (RM-ODP) provides an approach to modelling (i.e., the ODP viewpoints), it does not provide a prescriptive methodology that can be followed in developing a system [19]. In [20], an object oriented methodology is defined for the development of distributed telecommunications services. The proposed methodology helps producing models conceptually consistent with the ODP reference model by taking benefit of the systematic development process advocated by software engineering methodologies. The methodology consists of steps, models defined at each step, and the transitions inside and between the steps. Moreover this research work shows the relationship between the steps of the software development process and the ODP viewpoints (Figure 2), of prime interest for our study.

III. Mapping between the Network Management Models and the ODP Viewpoints

In section II.1.6., we have shown how the network management viewpoints take place in the software development process. In section II.2.1., we have defined the interrelations between the steps of the software engineering methodologies and the ODP viewpoints. Therefore, the ODP view of network management (i.e., the mapping betwecn Nctwork management models and ODP viewpoints) is straightforward (Figure 3 ).

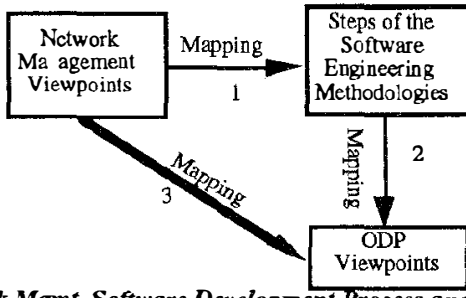

Fig. 3: Network Mgmt, Software Development Process and ODP relations This emphasis shows what aspect of network management is considered at each ODP viewpoint to enable the management of distributed systems (Figure 4). Moreover, specification techniques $/$ languages arc proposed in each viewpoint.

III.1. Enterprise Viewpoint of Network Management The objective of an enterprise specification is to characterize the requirements of the ODP system for both the client and provider's perspective, the interactions between the system and the environment and the policies of the system.

The management layers where management occurs should be selected from the set of management layers \{business, service, network and network element .

Moreover, the organizational model needs to be described. It consists of a set of management entities organized within the management architecture. This model can be described using a graphical representation such as OMT (Object Modeling Technique) [25].

The policies are derived from the goals of management and define the desired behavior of distributed heterogeneous networks and systems [21]. The goals of management are expressed by the management functional areas. A management policy specifies a relationship between a subject set of managers and a target set of managed objects which either obligates or authorises managers to perform activities on the managed objects [22]. In [22], a policy template bas been defined which identifies the essential characteristics of policies. This template is reused within our work to specify thc functional model of network management.
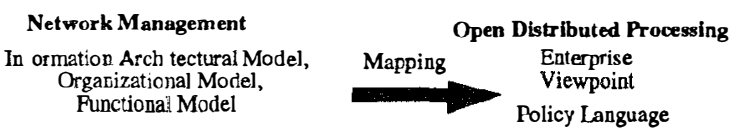

III.2. Information Vlewpoint of Network Management An information specification is a description of a structure that models the information in a system (domain of discourse, or problem domain) in terms of information bearing entities, relationships betwecn the entities, and constraints and rules that govern their behavior including creation and deletion.

For the information specification, both textual and graphical notations should be provided with a balance between these. Since OSI management has already standardized semi-formal notations for management information modeling, they are reused within the ODP information viewpoint. These are GDMO (Guidelines for the Definition of Managed Objects) [23] and GRM (General Relationship Model) [24] both following an object oriented approach. The (GDMO standard provides a set of templates to specify managed object classes. The GRM standard provides general and flexible relationship modcling mechanisms to specify n-ary relationships among managed objects.

For the graphical notation, the OMT object modeling tcchnique has been selected [25].

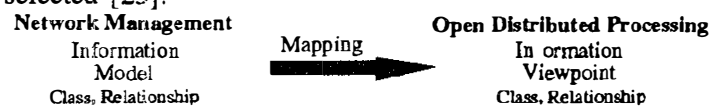

III.3. Computation Vlewpoint of Network Mgmt

The ODP computation viewpoint describes the objects within a distributed system and their interactions. In the computation viewpoint, a distributed application consists of a collection of interacting computational objects. A computational object provides a set of services that can be used by other objects. To enable other objects to access its services, an object offers computational interfaces, which are the only means by which other objects can use the services. The network management model that is related to this viewpoint is the functional architectural model. This latter consists of function blocks and reference points between these. A function block can be represented by one or several computational objects and a reference point can be supported by onc or several operational interfaces [26]. Note that managed object classes constituting the management information model and identified at the information viewpoint are encapsulated within computational objects. Indeed the information model should be exhausitive enough to support any of the management functions. Therefore it provides all information required to implement management functions. These functions are developed at the computation viewpoint and expressed via computational interfaces. A computational object is either a manager or a managed resource (agent in OSI terms), but the agent functionality is part of the engineering viewpoint.

For the graphical notation of the computation viewpoint, the notation proposed in the PRISM RACE II project [27] is reused. For the formal specification of the computational objects and their interfaces, the IDL (Interface Definition Language) notations [28] are used. In [29], the translation of GDMO templates into IDL interface templates is proposed.

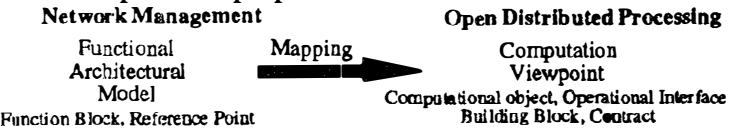

III.4. Engineering Viewpoint of Network Mgmt

The engineering viewpoint concentrates on the system components necessary to support the desired computational environment. 
If ODP conformant distributed platforms providing distribution transparency mcchanisms required for network management are available, the computation viewpoint can be directly implemented. The representation of a computational object (manager, managed resource) in the engineering viewpoint is the engineering computational object.

In terms of necessary requirements, the engineering viewpoint should describe the functionality needed to provide communications between the engineering computational objects, by means of a communication model which corresponds to the concept of channel in ODP.

A channel is not an object but a configuration concept, that consists of three types of enginccring objects of importance for OSI systems management: stub, binder and protocol objects [2]. All these objects implement some aspects of the agent functionalities. A stub object provides adaptation functions to support interaction between interfaces in different computing nodes. A binder manages the end-to-end integrity and QoS of the channel. A protocol object assures that computational objects can interact remotely by means of a management protocol.

Other aspects of the agent functionality such as creation and deletion of managed objects, notification dissemination, scoping filtering, etc., should be handled by supplementary engineering objects [30].

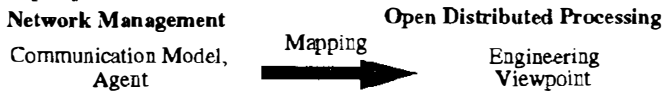

III.5. Technology Viewpoint of Network Management The technology viewpoint identifies the specific technologies used to realize the engineering components. At this level, the protocol used to convey management information may be CMIP, SNMP or SNMPv2. The engineering computational objects may be realized by the physical architectural model consisting of physical entities and their interfaces.

Network Management
$\begin{gathered}\text { Open Distributed Processing } \\ \text { Physical Architectural Model, Mapping } \\ \text { Management Protocol }\end{gathered}$
$\begin{gathered}\text { Technology } \\ \text { Viewpoint }\end{gathered}$

III.6. Summary

To summarize, figure 5 presents the different formalisms used to specify the network management models within the ODP framework. The engineering viewpoint is not tackled since we consider the use of a distributed system that handles this viewpoint transparently.

\section{Application to Connection Management}

The purpose of the connection management function (subfunction of configuration management) is to support distributed applications ranging from the simple telephony services to multimedia applications with their needs for connections. In this section, the five ODP viewpoints based on the network management models will be applied to cover the realization of the application from requirements to implementation.

\section{IV.1. Enterprise Viewpoint}

The different actors taking place in the connection management architecture are the user that requests a service, the service provider offering service sessions to users, and the network provider whose responsibility is to provide connections (Figure 6).

For the support of the connection management function, the service provider consists of a service session manager and a virtual session connection manager.

The service session manager provides the functionalities needed to create, delete and modify service sessions. A service session is the context supporting the activities and the execution of a service. The virtual session connection manager provides the functionalities needed to handle and control the communication resources. It controls the connectivity and the allocation of resources related to the session connections. A session connection is the context supporting the activities and functions for establishing connections related to a service session between users. A service session is oriented towards users while session connections are oriented towards user terminals.

For the support of the connection management function, the network provider consists of a network connection manager and a set of element connection managers. If several administrive domains have to be crossed to connect the users, several network connection managers may be present, one for each administrative domain.

The network connection manager provides interconnection of termination points of a network. The setup of the connection may span several domains. To provide its functionalities, the network connection manager relies on the element connection managers.

The element connection manager provides the connection of termination points inside the network element. It can satisfy requests to establish, modify, and release connections in its network element. These connections can be point-to-point unidirectional and bidirectional or point to multipoint unidirectional.

The network management organization is illustrated in figure 7 using OMT notations.

Connection management policies express in terms of rules what the system has to do to achieve given goals. Examples of policies using the policy template language defined in [22] are given bereafter.

\begin{tabular}{|l|c|c|c|c|}
\hline Subject & Modality & Activity & Target & Constraints \\
\hline The uses & may & change & $\begin{array}{l}\text { the QoS of } \\
\text { each flow type }\end{array}$ & if he is the owner of the service session. \\
\hline The user & may & join & the session & if he has already subscribed to the service \\
\hline
\end{tabular}

\section{IV.2. Information Viewpoint}

The information viewpoint deals with the specification of a network management information model at each management layer where connection management is involved, i.e., scrvice, network and element management layers. Management information means the information that is used to define how to manage particular types of resources. The amount of information that must be managed requires that a structured approach be taken to represent that information. Since many network entities share common characteristics, a unified way needs to be found to exploit this characteristic in order to maximize commonality and minimize implementation efforts and diversity. "Structured" calls for a model. "Commonality" requires a model that is applicable to all network entities. Therefore, a generic network management information model is the means to have a common model. In [31], such a generic model has been proposed, and is applicable to all management layers. It consists of three main components, namely, topology, connectivity and service. It is reused within our work and applied to the service, network and element management layers. Note that IINA-C, ETSI and ITU-T also propose generic models but none of them is applicable to all layers.

IV.2.1. Service Management Information Model for Connection Management

To administrate (i.e., monitor, control and manage) applications on an individual basis, a service information model is present for each application session. Every service model represents the configuration of the application session in terms of nodes and links between nodes. At the logical service view (Figure 8), nodes are users and links are flows exchanged among users with their associated Quality of Service (QoS). A QoS is defined for each flow type. At the physical service view, nodes are terminals and links are connections associated to the session. The logical service view deals with application sessions while the physical service view is related to end-to-cnd connections associated to sessions. 
The logical view is provided by the service session manager to the user. This perspective provides a means to the end-users to dynamically monitor, and manage their applications, while this has been for the long time a task performed by the network and systems managers. The physical view makes the link between the service and the network.

IV.2.2. Network Management Information Model for Connection Management

At the network management layer, the information model describes the end to end connectivity (Figure 8). This view is used to represent a global visibility of the network. Trail, connection and subnetwork concepts are present, independent of the underlying technology. The network management information model contains the managed network elements.

IV.2.3. Element Management Information Model for Connection Management

The element management information model represents a network element within the network that performs managed element functions, i.e., provides support and / or service to the user. At this level the information model must follow a standard (e.g., M3100 [12]) in order to hide the heterogeneity of existing proprietary MIBs providing management information concerning the network element.

IV.2.4. Network Element Information Model for Connection Management

The network element layer is inherently dependent on the technologies and architectures used in the specific switching and transmission equipment resources. At this layer, proprictary Management Information Bases (MIBs) of ATM switches are present. They provide all information required to manage specific equipment resources that provide network element layer functions.

Figure 8 represents the different models at all management layers informally.

\section{IV.3. Computation Viewpoint}

A computational object type diagram is now developed showing how computational objects interact to provide the connection management functionality (Figure 9). The computational model consists of one service session manager, one virtual session connection manager, one network connection manager, a set of element connection managers and a set of managed ATM switches computational objects. The network connection manager interacts with the set of element connection managers, while each element connection manager interacts with its corresponding managed ATM switch.

The information model at the information viewpoint provides all necessary information to support any management function, while the computational model performs a specific management function; in this work it is the connection management function.

The interfaces offered and required by the different computational objects are presented in the following computational object type diagram (Figure 9). Moreover, these interfaces may be specified individually in a more precise and formal way through the use of textual IDL templates.

The connection management function is currently under development using DCE (Distributed Computing Environment). Although this platform is far from fully realizing the promises of ODP, it has a quite similar computational model. Using DCE, the designer can clearly separate the telecommunication specific concerns from rather general issues in distributed processing. Since the DCE platform provides the distribution support, the application does not require any engineering specification. As a consequence, the connection management distributed application specified at the enterprise, information and computation viewpoints can be deployed and implemented in a quite straightforward way on DCE.

The Technology viewpoint, specifies how the system is implemented using hardware and software selected from available products. The implementation environment includes HP workstations running HP UX 9.1. We use the HP DCE 1.0.2 environment as distributed system and the $C$ language for programming the application components. As customer primises networks, we use Fore ASX 200 ATM Switches. Moreover, an access to the Swiss public ATM pilot is available.

\section{Conclusion}

In this paper, we have proposed an approach to the realization of the management of distributed systems within the ODP framework. A mapping between network management models structuring the network management complexity and the ODP viewpoints has been defined for that purpose. To pass from the proposed concepts to their application, emphasis has been put on the development of a connection management function. This application is currently under development in our laboratory using DCE as distributed processing environment and an end-to-end ATM infrastructure as communication platform

References

(1] S. Zuaty, J. Sclavos. "The Network Management Viewpoints: A New Way of Encompassing the Network Management Complexity". ACM Computer Comm. Review, July 94 . Casic Reference Model for ODP - Part 1: Overview and guide to use, ITUT Rec. X.901, Nov 93

Basic Reference Model for Open Distributed Processing - Part 2 Descriptive Model, ITU-T Draft Rec. X.902, 94 Basic Reference Model for ODP - Part 3: Prescriptive Model, ITU-T Draft Rec. X.903, Feb 94.

ISONEC 10040/TTU-T Rec. X.701: Systems Management Overview, 92. ITU-T Rec. M.3010: Principles for a Telecommunications Management Network, 93.

NMForum. "Discovering OMNIPoint". Prentice Hall, 93.

S. Aidarous, T. Pleviak. "TMN Into the 21st Century", 94, pp 19-71. W.J. Barr, T. Boyd, Y. Inoue. "The TINA Initiative". IEEE Comm. Mag. March 93.

SO 7498-4. Information Processing Systems - Open Systems Interconnection. Part4: Management framework, 89.

ISO/EC 10165-1/ ITU Rec. X720. "Management Information Model", 92.

[12] ITU-T Rec. M.3100: Generic Network Information Model, 93.

[13] EISI Draft Rec. "TMN Network Level View Managed Objects", Sept 94.

[14] M. Wakano et al. "Information Model to Support TINA Service and Management Applications", Globecom'94. [15] M. Rose, K. McCloghrie. "Management Information Base for Network Management of TCP/IP-Based Internets:MIB-II". RFC 1213, March 91.

[16] ISO 9596. Information Processing Systems. Open Systems Interconnection - Commion Management Information Protocol Specification, Jan 90.

[17] J.Case et al. "A Simple Network Management Protocol". RFC 1067, Aug 88.

[18] J. Case et al. "Protocol Operations for version 2 of the Simple Network Management Protocol (SNMPv2)". RFC 1448, April 93.

J-M. Cornily et al. "Guidelines for the Specification of Transport Network Management Services using the Reference Model for ODP". TINA'95, pp $445-460$.

J-P. Gaspoz. "Methodology for the Development of Distributed Telecommunications Services". Submitted for Publication to J. of Software

[21] R. Wies. "Policies in Nel.work and Systems Management - Formal Definition and Architecture". J. of Network and Systems Management, 94, $\mathrm{pp} 63-83$.

[22] X. Becker et al. "Domain and Policy Service Specification". IDSM Deliverable D6, SysMan Deliverable MA2V2, Oct 93

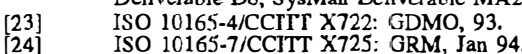
[25] I. Rumbaugh et al. "Object Oriented Modeling and Design". Prentice Hall,

[26] A. Bridge, H. Katz. "TMN Evolution towards TINA". IEEE NOMS'94, pp $278-286$

P. McLaughlin, W. Donnely. "ODP as a unifying Framework for the Development of Co-operative Service Management Systems". Proc. of TINA'95, pp 13-28.

[28] OMG. "The Common Object Request Broker: Architecture and Specification". Dec 93.

[29] Joint Xopen/NM Fonum. Translation of GDMO/ASN.1 Specifications into CORBA-IDL. Draft 0.02 , July 94 .

[30] ESMA Draft. Contribution to ODP Distributed Management: The Extended Systems Management Architecture Related to ODP, May 94.

[31] J.P. Gaspoz. "Obiect Oriented Method and Architecture for VPN Service 


\begin{tabular}{|c|c|c|c|c|c|}
\hline Viewpoints & Requirements & Analysis & $\begin{array}{c}\text { High } \\
\text { Leyel } \\
\text { Design }\end{array}$ & $\begin{array}{l}\text { Deta:led } \\
\text { Design }\end{array}$ & Implemedtation \\
\hline Enterprise & & & & & \\
\hline Information & & & & & \\
\hline Computation & & & & & \\
\hline Eugiveetriug & & & & & \\
\hline Technology & & & & & ২ \\
\hline
\end{tabular}

Figure 2: Steps of the Software Development Process versus ODP Viewpoints

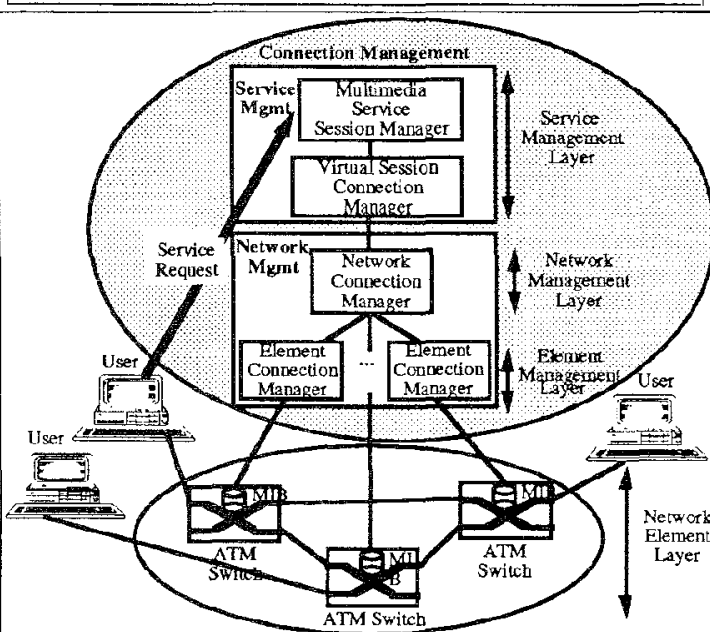

Figure 6: The Overall Architecture of the Connection Management Function

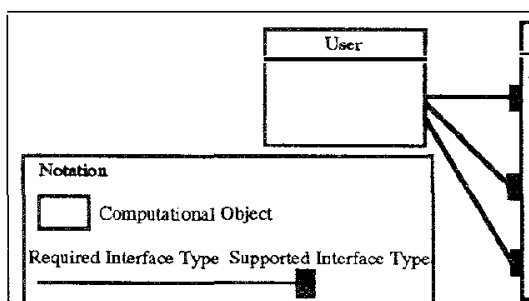

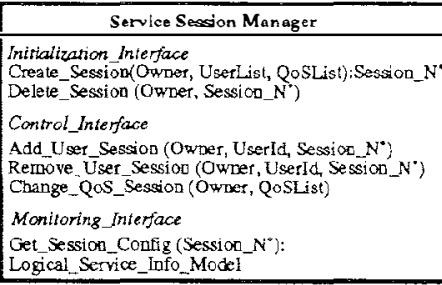

Network Connection Manager Initialization_Interface SetupSubnetworkConn (In_SN_TPId, Out_SN_TPIC, directionality $Q\left(C^{\circ}\right)$ : SN_Connection_Id

Control_Interface

Altach (SN_TP_Id)

Moniloring_Interfuce

SN_Connectiou_Status (Connection_Id):

SN_Connection_Entry

GTP: Grolp Termination Point: TP. Termination Poim SN: Subnetwork: QoC: Quatity of Conmection

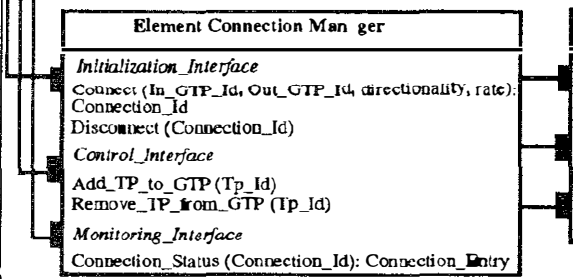

Virtual Session Connection Manager

\section{Initialization_Interface}

Create_Session_Conn (Terminal_Add _l ist,

Qodist): Session_Con__

Dession Conn (Session_Conn_N

Control_Interface

Add_User_Sessien (Terminal_Addr, Sessi $\bullet$ _COnn_N*) Change_QOC_Session_Conn $(\mathrm{Q} \bullet \mathrm{SL}$. is $)$

Monitoring_Interface

Get_Session_Coon_Config (Session_Conn_N*):

Info_Muck

C: Quality of Connection

\begin{tabular}{|c|}
\hline Managed ATM Switch \\
\hline 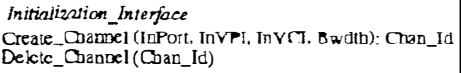 \\
\hline $\begin{array}{l}\text { Control_Snterface } \\
\text { Set_Cban_Bandwidth (Bandwidth) }\end{array}$ \\
\hline $\begin{array}{l}\text { Monitoring_Interface } \\
\text { Coan_Scatus (Cran_Id): Chan_Eotry }\end{array}$ \\
\hline
\end{tabular}

GTP: Group Termination Point; TP. Terminatiou Point

Figure 9: The computational object type diagram for Connection Management

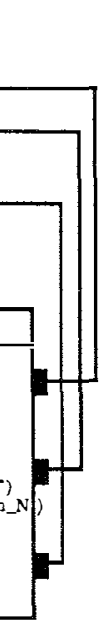

Notations

.

Poit

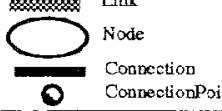

formation Model

at each Management Layer
Network Management Models

Mappings

ODP Viewpoints

Lead to

Realization of :

the Management of Distributed Applications, Distributed Management Applications

Figure 4: Mapping between NM Models and ODP Viewpoints

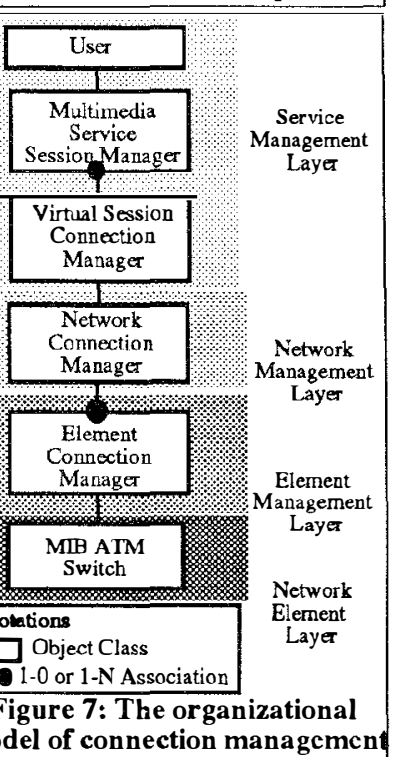
model of connection management

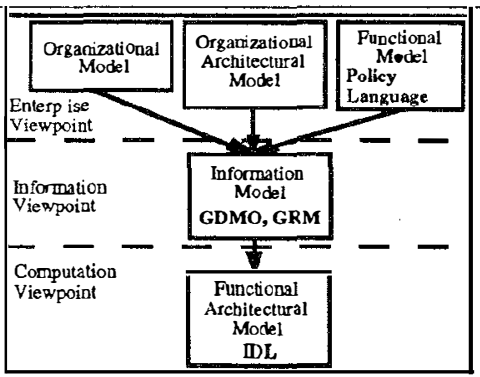

Fig. 5: Notations for the Formalism of Network Management Models in the ODP Framework

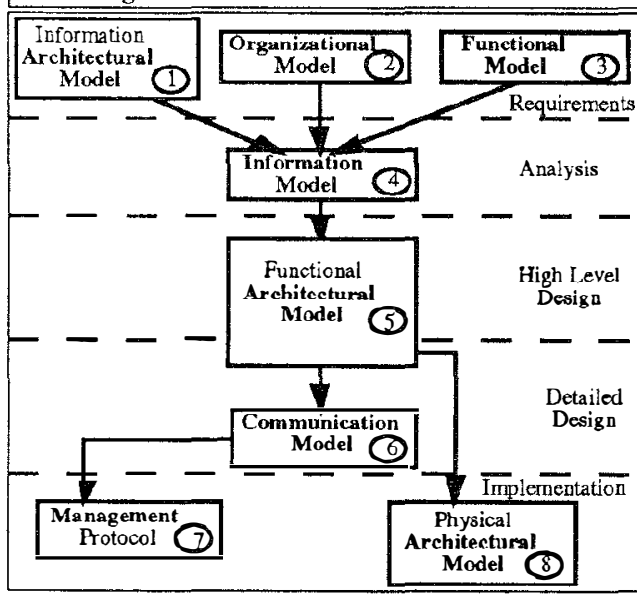

Figure 1: The Network Management Models within the Softwarc Development Process 